\title{
Built-in Starter with Pulse Cut-out for HID Lamps
}

\author{
Shunichi SASAKI*, Takenobu IIDA* and Russell LOANE** \\ * : Iwasaki Electric Co.,Ltd. Saitama Plant \\ 1-1 Ichiriyama machi Gyoda city Saitama pref. 361 Japan \\ ** : Eye Lighting Industries Pty Limited \\ 157 Wellington Road, East Brisbane, Qld4169, Australia
}

Received June 12, 1996

\section{ABSTRACT}

Recently, High Pressure Sodium lamps (H. P. S. L.) and Metal Halide lamps (M. H. L.) have been operated on Mercury Vapour lamp ballasts. But for both H.P.S.L. and M.H.L. it is necessary to use a starter to ignite the lamp. The usual starter can create some problems being radio frequency noise generation, insulation failure and others caused by continuous pulsing at the point of lamp failure. One of the solutions to the above problems is to cut out the starting pulse when the lamp fails. This paper shows a new built-in starter for such lamps that can cut out the starting pulse when the lamp fails. This new built-in starter is constructed by a Ferro-Electric Capacitor (FEC) ${ }^{1,2)}$ which has nonlinearity characteristics, a semi-conductor switching device (SIDAC) and some resistors. This starter can generate the starting pulse using the FEC's non-linearity characteristics and also can cut out the pulse by means of losing its characteristics above Curie point. And for H.P.S. lamps, in the case of arc tube leakage into the vacuum outer jacket, an arcing will be formed on the FEC's surface by the starting pulse. Then the FEC will be fired, and the pulse will be cut out permanently.

\section{Introduction}

Mercury vapour lamps (M.V.L.), although relatively inexpensive, lack the high efficacy of High Pressure Sodium lamps (H.P.S.L.) and the improved colour quality of Metal Halide lamps (M.H.L.). Many users therefore have chosen to use H.P.S.L. and M.H.L. instead of M.V.L.

For both H.P.S.L. and M.H.L. it is necessary to use a starter to ignite the lamp. The starter can be either an external or an internal (built-in) type.

The presence of a starter can create some problems at the point of lamp failure. For example;

1) in the case of lamp starting failure:

* radio frequency noise generation

* insulation failure in the circuit

2 ) in the case of the arc tube leakage in the vacuum outer jacket as H.P.S.L. :

* abnormal arcing in the outer jacket

* abnormal arc current

These phenomena may cause ballast failure.

One of the solutions to the above problems is to cut out the starting pulse when the lamp fails.

\section{Structure and Characteristics of Internal Starters}

The internal starter has been widely employed in H.P.S.L. and M.H.L. in Japan to enable them to be operated on a M.V.L. ballast.

The functions required of the lamp starter can be summarized as follows.
1) A pulse voltage (energy) sufficient to start the lamp must be generated.

2) Pulse generation must be cease immediately after the lamp starts.

3) Pulse voltage generation must be cut out hen the lamp fails.

But many existing internal starters are a mechanical type (thermal switch) and generate a pulse voltage which is not controlled in peak voltage, width and phase. This can result in the requirement for special insulation to the ballast.

In preparation for lamp failure, it is desirable to provide a cut out function for the pulse generation with a timer, as is done with some external starters. However, it is very difficult to equip the conventional mechanical internal starter with such a pulse cut-out function.

We therefore decided to employ an electronic internal starter which has similar characteristics to an external starter. This starter can provide complete control of the starting pulse and in the case of lamp failure or fault, will cut out the pulse automatically.

The internal starter we have employed consists of a series circuit of a non linear ceramic capacitor and a semiconductor switch.

Fig. 1 is a schematic diagram showing changes in the voltage and dielectric polarization (hysteresis locus) ${ }^{3)}$ when an a.c. voltage is applied to the non linear cermic capacitor. 


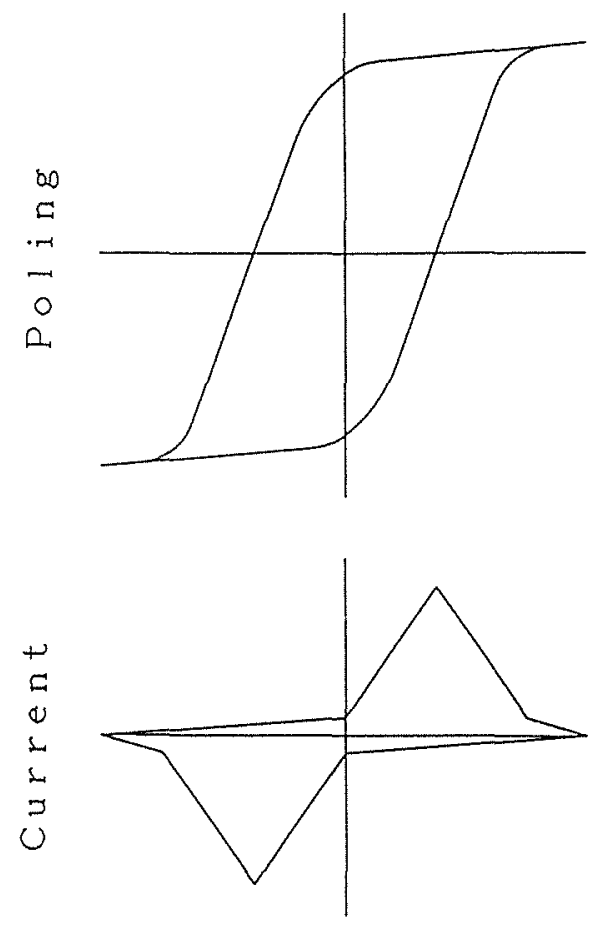

Electric Field

Fig. 1 Schematic diagrams of hysteresis locus on poling and current change for non-linear ceramic capacitor.

Fig. 1 indicates that a large current change occurs when the polarization reverses. In detail, the maximum change of current $(\mathrm{dI} / \mathrm{dt})$ occurs at the time of polarization reversal that occurs every half a cycle of the a.c. voltage.

When an inductive ballast is connected to it in series, the pulse voltage $(\mathrm{Vp})$ is generated in combination with its inductance component (L) as expressed by the following formula.

$$
\mathrm{Vp}=-\mathrm{L}(\mathrm{d} \mathrm{I} / \mathrm{dt})
$$

The semiconductor switch (SIDAC*), connected in series to the non linear ceramic capacitor, is designed to advance the time change of current and is capable of generating a higher pulse voltage.

* : Produced by Shindengen Electric Mfg. Co.,Ltd.

For the non linear ceramic capacitor to be employed in the starter, we have developed a new ceramic capacitor of a barium titanate type. ${ }^{1)}$ Fig. 2 shows the temperature characteristics of the dielectric constant. It becomes a ferroelectric substance at a temperature below the Curie Point and acquires the non linear characteristics shown in Fig. 1.

At a temperature above the Curie Point, it becomes a paraelectric substance and loses the non linear characteristics. In this state it becomes impossible to generate a pulse voltage. In the following explanation, the non linear capacitor that we use as the internal starter is referred to as the FEC (Ferro Electric Capacitor) and the starter employing the FEC is referred to as the FEC starter.

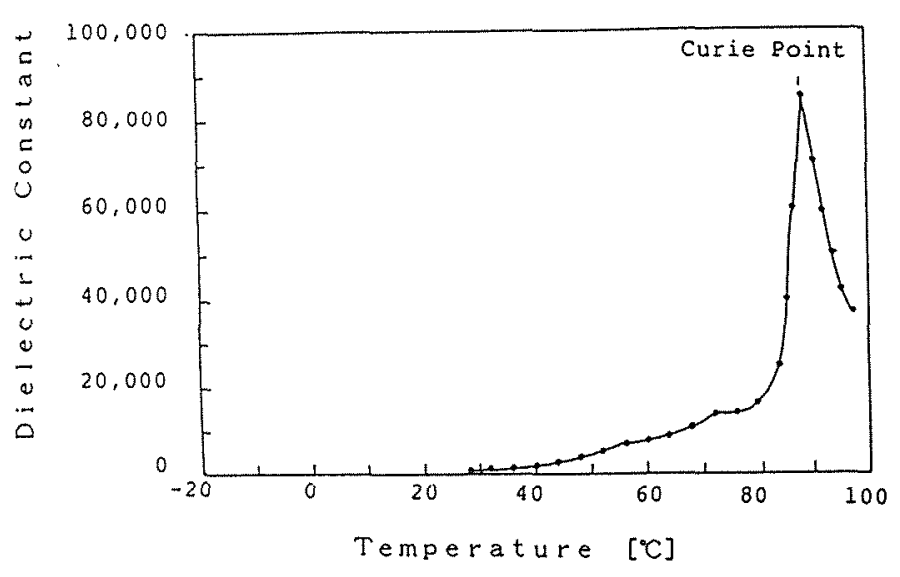

Fig.2 Dielectric constant vs. Temperature for the new ceramic capacitor.

\section{Circuit Structure and Characteristics of the FEC Starter}

The circuit structure of the FEC starter built in a H.P.S.L. is shown in Fig. 3-(a). The circuit structure of the FEC starter built in the M.H.L. is shown in Fig. 3-(b).

In Fig. 3-(a) and (b), the Rs resistance acts to keep constant the pulse phase and peak voltage value generated at every half a cycle of the a.c. voltage. The Rc resistance acts to discharge the pyroelectricity generated when FEC temperature rises above the Curie Point after the lamp has started, thus preventing deterioration of the characteristics of the FEC elements. The bimetal switch, inserted in series to the FEC starter, is opened by the heat generated from the arc tube after the lamp starts so as to cut out the generation of pulse voltage, and at the same time, prevent deterioration characteristics by keeping the potential off the starter while the lamp is lit.

Fig. 4-(a) and (b) show the pulse voltage wave forms that are generated when each starter is connected to a $250 \mathrm{w}$ mercury lamp ballast.

\section{Lamp Abnormal Phenomena and Countermeasures}

Abnormal phenomena of H.P.S.L. and M.H.L. can largely be summarized as follows.

1) Toward the end of the lamp service life, starting of the arc tube becomes more difficult until eventually the lamp will not ignite.

Consequently, the starter continues to generate the pulse voltage, causing radio frequency noise and insulation failure in the starter and the lighting circuit.

2) With lamps having a vacuum outer jacket, such as H.P.S.L., if the arc tube leaks near the end of the service life of the lamp, the lamp may start at a lower 

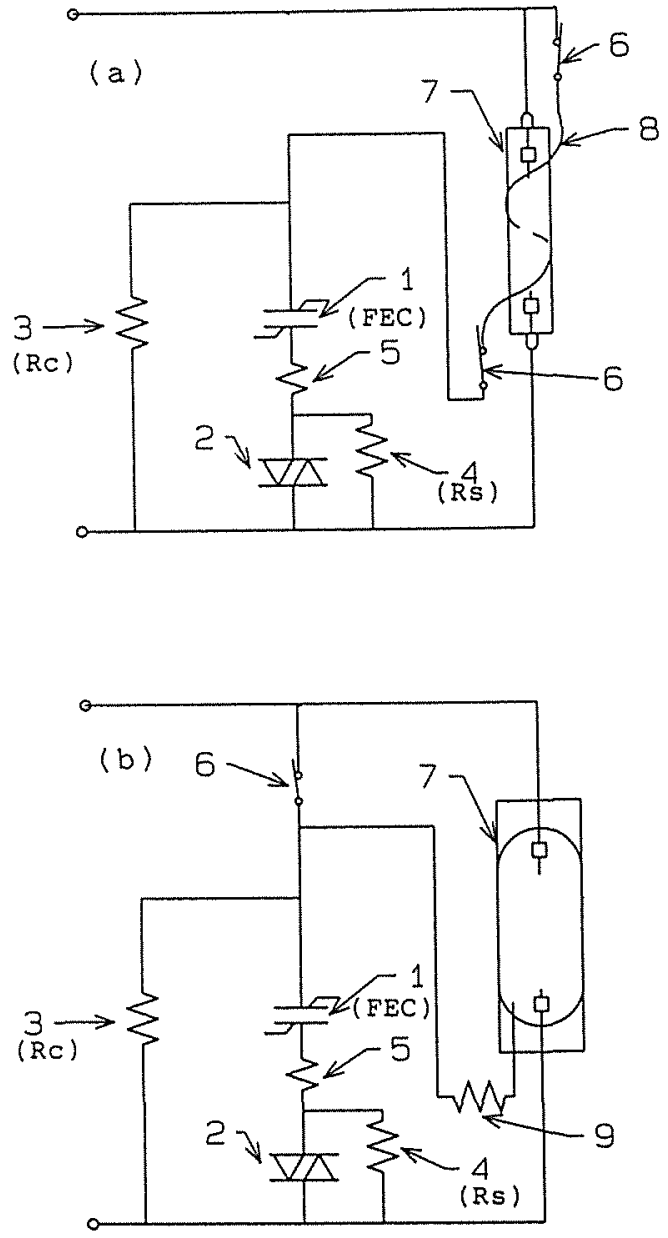

Fig. 3 Schematic diagrams of H.P.S.L.(a) and M.H.L.(b) with the FEC starter. 1:FEC 2:SIDAC 3:Rc resistor 4:Rs resistor 5 :Current damper 6:Bimetal Sw. 7:Arc tube 8:Starting accelerator $9:$ Starting resistor

lamp voltage or discharge may occur inside the outer jacket. If the lamp remains lit at a lower lamp voltage, an over-current keeps flowing to the ballast.

If a discharge occurs inside the outer jacket, a very large current will flow to the ballast momentarily.

Both situations are a cause of ballast failure.

Countermeasures to prevent the above abnormal phenomena include;

a. When the lamp will no longer start, the pulse generation will cut out after a certain time.

b. If leakage occurs in a lamp which has a vacuum outer jacket, the starter should detect such leakage and then cut out pulse generation.
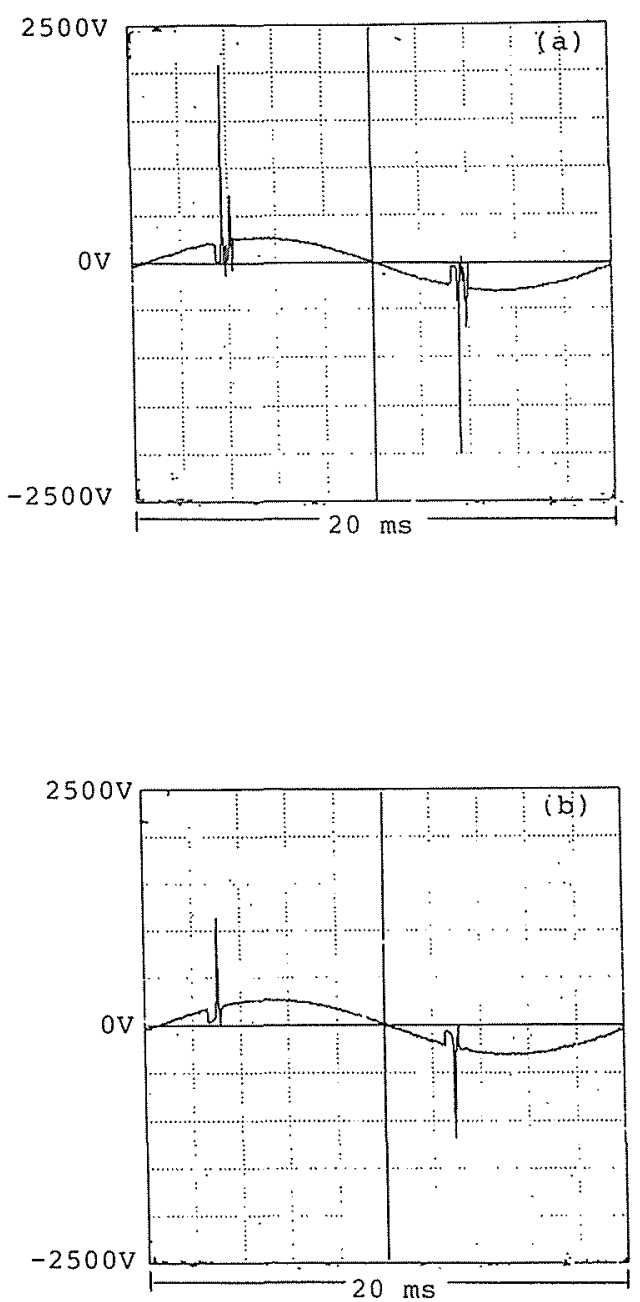

Fig. 4 Pulse profiles generated by the FEC starter for H.P.S.L.(a) and M.H.L.(b) combined with M.V.L. $250 \mathrm{~W}$ ballast.

\section{Principles of the Pulse Cut-out}

Pulse Cut-out Function 1

If the FEC temperature rises when the lamp does not start, it will be able to cut out the pulse generation.

The reason for this is as follows. When FEC temperature exceeds about $60^{\circ} \mathrm{C}$, the pulse voltage drops rapidly and at a temperature above $90^{\circ} \mathrm{C}$, no pulse will be generated.

The Rc resistor shown in the circuit diagrams (Fig. 3-a,b) of the FEC starter is an element installed to discharge pyroelectricity. However when the lamp does not start, this element works as a heater, too. The current continues to flow to this element. Although this current is very small, less than $10 \mathrm{~mA}$, it is capable of heating the resistance element and the FEC connected to it as shown in Photograph-1. 


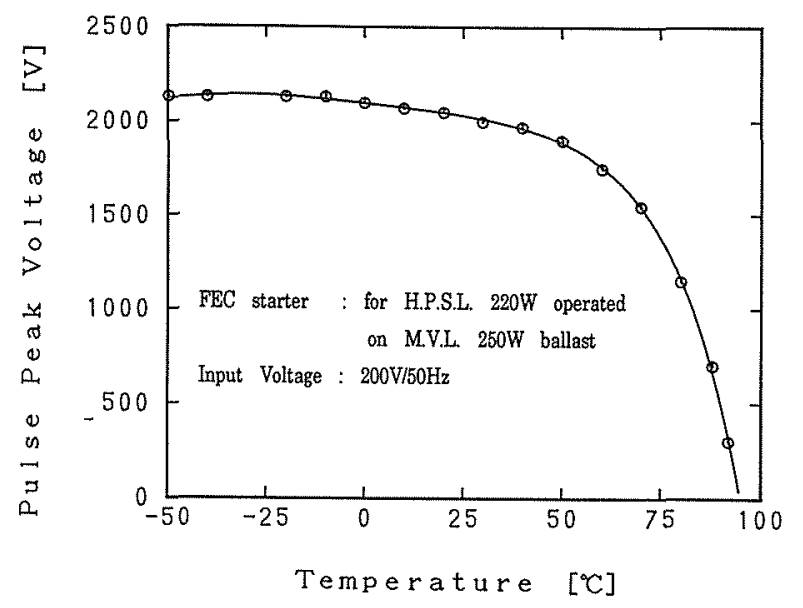

Fig. 5 The relation between FEC temperature and pulse peak voltage.

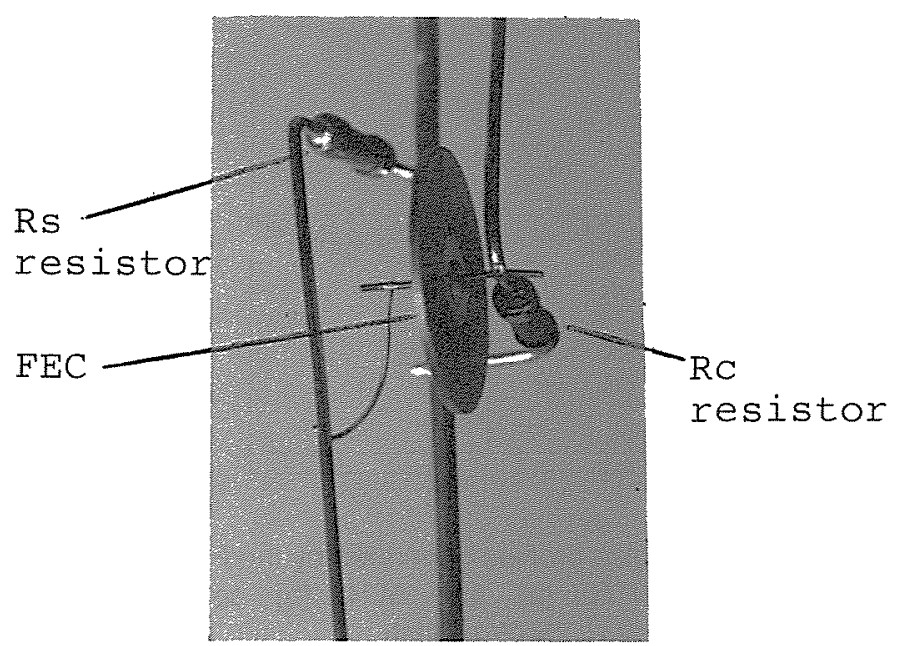

Photograph-1 Location of FEC, Rc resistor and Rs resistor in a HPS lamp.

Fig. 6 shows a temperature rise curve of the FEC's terminal lead when the lamp does not start. If the FEC temperature exceeds the Curie Point of $90^{\circ} \mathrm{C}$, due to heating of the resistance element, the FEC becomes incapable of generating the pulse.

The speed at which the FEC temperature rises depends on the resistance, size of the resistance element, and the distance between the FEC and the resistance element. By combining these factors suitably, it is possible to obtain an appropriate temperature rise speed. When ambient temperature is normal, the pulse can be cut out in 3 to 5 minutes as shown in Fig. 7.

\section{Pulse Cut-out Function 2}

With lamps having a vacuum outer jacket, such as H.P.S.L., if the arc tube leaks, the insulation inside the outer jacket becomes poor. If the lamp incorporates the FEC

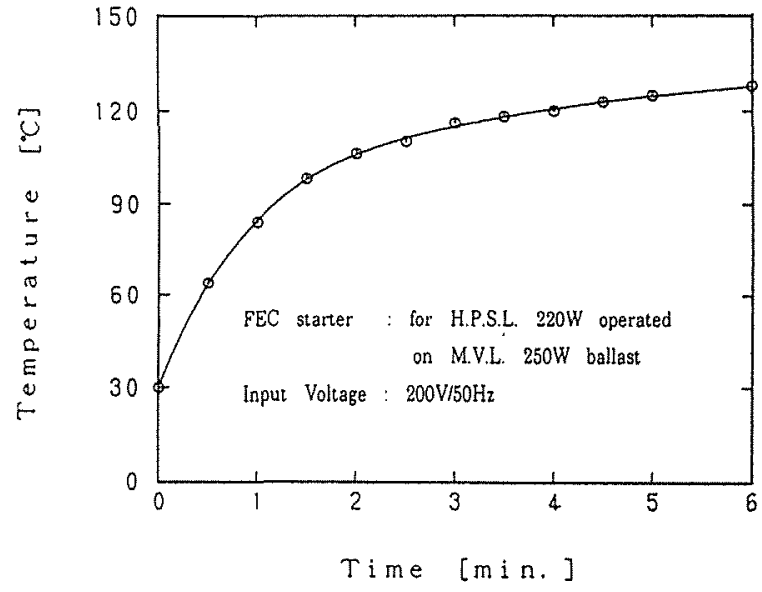

Fig. 6 Temperature rising curve at the FEC's terminal lead when the lamp does not start. FEC: diameter $=18 \mathrm{~mm}$, thickness $=0.7 \mathrm{~mm}$ Rc resistor: resistance $=80 \mathrm{k} \Omega$

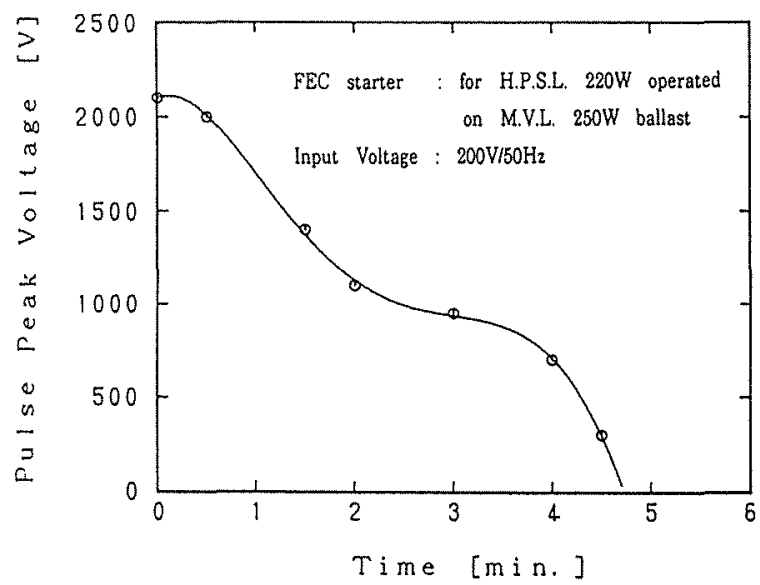

Fig. 7 Decrease in the generating pulse voltage when the lamp does not start.

starter, the pulse voltage generated by the starter breaks the insulation between the electrodes on the both sides of the FEC, causing discharge at the edges of the FEC, because the thickness of FEC is very thin, less than $1 \mathrm{~mm}$. This discharge, in turn, destroys the FEC itself, thus cutting out further pulse generation.

In order to prevent continuous flow of a large current due to discharge between the sides of the FEC, the FEC is provided with a current damper as a filament.

\section{Conclusion}

By employing the correct types of the FEC, SIDAC and resistance elements in the starter circuit within a lamp, and ensuring their appropriate interaction, it has become possible to generate a pulse voltage at an optimum level to start the lamp and at the same time provide a function to cut 
out the generation of pulse voltage in the case of lamp failure.

This development has achieved a substantial reduction in radio frequency noise generated from lamps and eliminated ballast and starting circuit failures almost entirely when a lamp failure occurs.

\section{References}

(1) T. lida et al.: Ceramic Pulse Generating Capacitor and Built-In Starter for High Pressure Sodium Lamps.

34th IES National Convention, Sydney, 1988 pp.5965

(2) S. Sasaki et al.: Metal Halide Lamps Designed for use with Standard High Pressure Sodium Ballast.

IES of Australia and New Zealand, Gold Coast, 1992 pp. $149-158$

(3) B. Jaffe, W.R.Cook Jr. And H. Jaffe : Piezoelectric Ceramics Academic Press London and New York 1971 p. 34 